\title{
On the $k^{\text {th }}$ Eigenvalues of Trees with Perfect Matchings
}

\author{
An Chang ${ }^{1}$ and Wai Chee Shiu $\|^{2}$ \\ ${ }^{1}$ Department of Mathematics, Fuzhou University, Fuzhou, Fujian, 350002, P.R. China. \\ ${ }^{2}$ Department of Mathematics, Hong Kong Baptist University, Kowloon Tong, Hong Kong, P.R. China.
}

received March 29, 2006, revised August 15, 2007, accepted November 2, 2007.

Let $\mathcal{T}_{2 p}^{+}$be the set of all trees on $2 p(p \geq 1)$ vertices with perfect matchings. In this paper, we prove that for any tree $T$ in $\mathcal{T}_{2 p}^{+}$, the $k$ th largest eigenvalue $\lambda_{k}(T)$ satisfies $\lambda_{k}(T) \leq \frac{1}{2}\left(\sqrt{\left\lceil\frac{p}{k}\right\rceil-1}+\sqrt{\left\lceil\frac{p}{k}\right\rceil+3}\right)(k=1,2, \ldots, p)$. This upper bound is known to be best possible when $k=1$. The set of trees obtained from a tree on $p$ vertices by joining a pendent vertex to each vertex of the tree is denoted by $\mathcal{T}_{2 p}^{*}$. We also prove that for any tree $T$ in $\mathcal{T}_{2 p}^{*}$, its $k$ th largest eigenvalue $\lambda_{k}(T)$ satisfies $\lambda_{k}(T) \leq \frac{1}{2}\left(\sqrt{\left\lfloor\frac{p}{k}\right\rfloor-1}+\sqrt{\left\lfloor\frac{p}{k}\right\rfloor+3}\right) \quad(k=1,2, \ldots, p)$ and show that this upper bound is best possible when $k=1$ or $p \not \equiv 0(\bmod k)$. We further give the following inequality

$$
\lambda_{k}^{*}(2 p)>\frac{1}{2}\left(\sqrt{t-1-\sqrt{\frac{k-1}{t-k}}}+\sqrt{t+3-\sqrt{\frac{k-1}{t-k}}}\right) \quad t=\left\lfloor\frac{p}{k}\right\rfloor,
$$

where $\lambda_{k}^{*}(2 p)$ is the maximum value of the $k$ th largest eigenvalue of the trees in $\mathcal{T}_{2 p}^{*}$. By this inequality, it is easy to see that the above upper bound on $\lambda_{k}(T)$ for $T \in \mathcal{T}_{2 p}^{*}$ turns out to be asymptotically tight when $p \equiv 0(\bmod k)$.

Keywords: tree, eigenvalue, perfect matching.

\section{Introduction}

Let $G$ be a simple graph, i.e., a graph without loops or multiple edges. Suppose the vertex set of $G$ is $V(G)=\left\{v_{1}, v_{2}, \ldots, v_{n}\right\}$. The adjacency matrix of $G$ is an $n \times n$ matrix $A(G)=\left(a_{i j}\right)$, where $a_{i j}=1$ if $v_{i}$ is adjacent to $v_{j}$, and $a_{i j}=0$ otherwise. The characteristic polynomial of $G$ is $\operatorname{det}(\lambda I-A(G))$, which is denoted by $P(G ; \lambda)$. Since $A(G)$ is symmetric, its eigenvalues are real; moreover, they are independent of the ordering of the vertices of $G$. As usual, we write them in non-increasing order as $\lambda_{1}(G) \geq \lambda_{2}(G) \geq \lambda_{3}(G) \geq \cdots \geq \lambda_{n}(G)$ and call them the eigenvalues of $G$. If $G$ is a bipartite graph, then $\lambda_{i}(G)=-\lambda_{n-i+1}(G)$ for $i=1,2, \ldots,\lfloor n / 2\rfloor$ (see [6]), where $\lfloor x\rfloor$ denotes the largest integer less than or equal to $x$, i.e., the floor function of $x$ when $x$ is a real number. Similarly, $\lceil x\rceil$ denotes the least integer greater than or equal to $x$, i.e., the ceiling function of $x$.

${ }^{\dagger}$ Corresponding author. The work was supported by the National Natural Science Foundation of China (No. 10371019 and 10431020); CERG Research Grant Council of Hong Kong; and Faculty Research Grant, Hong Kong Baptist University.

1365-8050 (C) 2007 Discrete Mathematics and Theoretical Computer Science (DMTCS), Nancy, France 
Two distinct edges in a graph $G$ incident with the same vertex will be called adjacent edges. A matching of $G$ is a set of edges in $G$ such that no two of them are adjacent. A largest matching is called a maximum matching. The cardinality of a maximum matching of $G$ is commonly known as its matching number, denoted by $\mu(G)$. Let $M$ be a matching of $G$. $M$ is called an s-matching of $G$ if $M$ contains exactly $s$ edges of $G$. A vertex $v \in V(G)$ is said to be $M$-saturated if it is incident with an edge of $M$, otherwise $v$ is called an $M$-unsaturated vertex. The matching $M$ of $G$ is called a perfect matching if all vertices of $G$ are $M$-saturated. Trees are connected acyclic graphs, and it is obvious that they are also bipartite graphs. So we only need to investigate those eigenvalues $\lambda_{k}(T)$ of a tree $T$ with $n$ vertices for $k=1,2, \ldots,\lfloor n / 2\rfloor$.

Throughout this paper, we denote by $\mathcal{T}_{n}$ and $\mathcal{T}_{2 p}^{+}$the set of trees on $n$ vertices and the set of trees on $2 p$ vertices with perfect matchings. For simplicity, a tree with $n$ vertices is often called a tree of order $n$. For symbols and concepts not defined in this paper we refer to the book [2].

The investigation on the eigenvalues of trees in $\mathcal{T}_{n}$ is one of the oldest problems in the spectral theory of graphs and has been intensively studied by many authors (see [1, 6, 11, 12, 13, 15]). A classic result is that for any $T \in \mathcal{T}_{n}, \lambda_{1}(T) \leq \sqrt{n-1}$ and equality holds if and only if $T$ is the star $K_{1, n-1}$. In particular, H. Yuan [12] studied the $k$ th eigenvalue of a tree $T \in \mathcal{T}_{n}$ and obtained the following upper bound.

Theorem $1.1([\mathbf{1 2}])$ Let $T$ be a tree in $\mathcal{T}_{n}$. Then

$$
\lambda_{k}(T) \leq \sqrt{\left\lfloor\frac{n-2}{k}\right\rfloor} \quad\left(2 \leq k \leq\left\lfloor\frac{n}{2}\right\rfloor\right)
$$

and the upper bound is best possible if $n \equiv 1(\bmod k)$.

J.Y. Shao [15] improved the above result.

Theorem 1.2 ([15]) Let $T$ be a tree in $\mathcal{T}_{n}$. Then

$$
\lambda_{k}(T) \leq \sqrt{\left\lfloor\frac{n}{k}\right\rfloor-1} \quad\left(1 \leq k \leq\left\lfloor\frac{n}{2}\right\rfloor\right) .
$$

Moreover, the bound is best possible when $n \not \equiv 0(\bmod k)$ and it is an asymptotically tight bound when $n \equiv 0(\bmod k)(2 \leq k \leq\lfloor n / 2\rfloor)$.

Concerning the trees in $\mathcal{T}_{2 p}^{+}$there are lots of results on the first two largest eigenvalues (see $\left[\begin{array}{ll|l|l|l|l|l|}3 & 4 & 5 & 8 & 9\end{array}\right.$ 10, 16, 17, 18]).

Frucht and Harary [7] gave the following construction of graphs. Given two graphs $G$ and $H$, the corona of $G$ with $H$, denoted by $G \odot H$, is the graph with

$$
\begin{aligned}
& V(G \odot H)=V(G) \cup \bigcup_{i \in V(G)} V\left(H_{i}\right), \\
& E(G \odot H)=E(G) \cup \bigcup_{i \in V(G)}\left(E\left(H_{i}\right) \cup\left\{i u_{i} \mid u_{i} \in V\left(H_{i}\right)\right\}\right),
\end{aligned}
$$

where $H_{i} \cong H$ for all $i \in V(G)$.

Let $T_{2 p}^{1}=K_{1, p-1} \odot N_{1}$ (see Fig. 1.1), where $N_{s}$ is the null graph (i.e., edgeless graph) of order $s$. G.H. Xu [17] got the following initial result. 


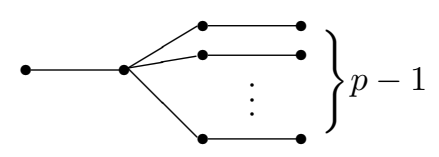

Fig. 1.1: The tree $T_{2 p}^{1}$
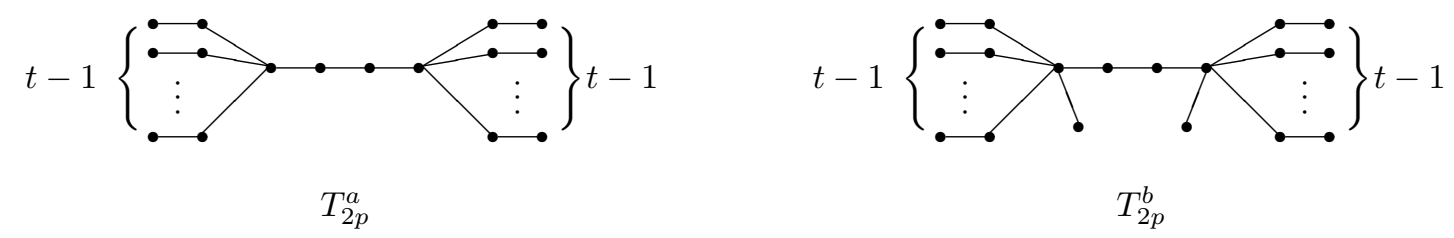

Fig. 1.2: Two graphs $T_{2 p}^{a}$ and $T_{2 p}^{b}\left(=T_{2 p}^{2^{\prime}}\right)$

Theorem 1.3 ([17]) Let $T$ be a tree in $\mathcal{T}_{2 p}^{+}$. Then

$$
\lambda_{1}(T) \leq \frac{1}{2}(\sqrt{p-1}+\sqrt{p+3})=\lambda_{1}\left(\mathcal{T}_{2 p}^{1}\right) \quad p=1,2,3, \ldots
$$

and equality holds if and only if $T \cong T_{2 p}^{1}$.

A. Chang [3] studied bounds for the second largest eigenvalue of trees in $\mathcal{T}_{2 p}^{+}$and proposed the following conjecture:

Let $p$ be a positive integer, and $T$ be a tree in $\mathcal{T}_{2 p}^{+}$. Then

$$
\lambda_{2}(T) \leq\left\{\begin{array}{ll}
r^{\prime} & \text { if } p=2 t \\
r^{\prime \prime} & \text { if } p=2 t+1
\end{array} \text { for } t=1,2,3, \ldots,\right.
$$

where $r^{\prime}$ and $r^{\prime \prime}$ are the maximum positive roots of the equations $x^{3}-(t+1) x+1=0$ and $x^{4}-(t+2) x^{2}+x+1=0$, respectively. Equality holds in the first inequality if and only if $T \cong T_{2 p}^{a}$, and equality holds in the second inequality if and only if $T \cong T_{2 p}^{b}$, where $T_{2 p}^{a}$ and $T_{2 p}^{b}$ are the trees shown in Fig. 1.2

More recently, J-M. Guo and S-W. Tan [9] proved that the second inequality holds but the first one does not hold. A correct version of the first inequality was given by J-M. Guo and S-W. Tan in [10]. Their results can be stated as follows. 


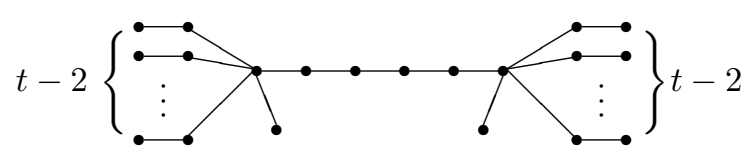

Fig. 1.3: The tree $T_{2 p}^{2}$

Theorem $1.4([\mathbf{9}, \mathbf{1 0}])$ Let $p$ be a positive integer, and $T$ be a tree in $\mathcal{T}_{2 p}^{+}$. Then

$$
\lambda_{2}(T) \leq\left\{\begin{array}{ll}
r_{1} & \text { if } p=2 t \\
r_{2} & \text { if } p=2 t+1
\end{array} \text { for } t=2,3, \ldots,\right.
$$

where $r_{1}$ and $r_{2}$ are the maximum positive roots of the equations $\left(x^{4}-(t+1) x^{2}+1\right)\left(x^{2}+x-1\right)+x=0$ and $x^{4}-(t+2) x^{2}+x+1=0$, respectively. Equality holds in the first inequality if and only if $T \cong T_{2 p}^{2}$, and equality holds in the second inequality if and only if $T \cong T_{2 p}^{2^{\prime}}$, where $T_{2 p}^{2}$ and $T_{2 p}^{2^{\prime}} \cong T_{2 p}^{b}$ are the trees shown in Fig. 1.3 and 1.2 respectively.

It is natural to consider the problem of determining upper and lower bounds of the $k$ th eigenvalues of the trees in $\mathcal{T}_{2 p}^{+}$. This is the purpose of our paper.

\section{Main results}

We need some groundwork before giving the main result. Before we recall the well-known Cauchy Interlacing Theorem [6. Theorem 0.10], we introduce some notation and terminology first. A vertex subset with $k$ vertices is called a $k$-vertex subset. Suppose $V^{\prime}$ is a subset of vertices. $G-V^{\prime}$ is the subgraph of $G$ obtained by deleting all vertices in $V^{\prime}$ together with their incident edges. Cauchy Interlacing Theorem usually plays an important role in the estimation of the $k$ th eigenvalue of trees.

Theorem 2.1 (Cauchy Interlacing Theorem) For every graph $G$ and every $k$-vertex subset $V^{\prime}$ we have

$$
\lambda_{i}(G) \geq \lambda_{i}\left(G-V^{\prime}\right) \geq \lambda_{i+k}(G), \quad i=1,2, \ldots, n-k .
$$

Lemma 2.2 ([1]) Let $G$ be a graph and $H$ a subgraph of $G$. Then $\lambda_{1}(H) \leq \lambda_{1}(G)$.

Lemma 2.3 ([15]) Let $T$ be a tree in $\mathcal{T}_{n}$. Then for any positive integer s, there exists a vertex $v \in V(T)$ such that the largest component of $T-v$ has order at most $\max \{n-1-s, s\}$ and all other components of $T-v$ have orders at most $s$.

It is worth mentioning that when the tree $T$ considered in Lemma 2.3 is in $\mathcal{T}_{2 p}^{+}$, i.e., $T$ is a tree with a perfect matching, then obviously all components of $T-v$ but one have perfect matchings. The only component without perfect matching, say $T_{0}$, has matching number $\mu\left(T_{0}\right)=\frac{1}{2}\left(V\left(T_{0}\right)-1\right)$, and the only unsaturated vertex of $T_{0}$ is the vertex $w$ which is adjacent with $v$ in $T$ and $w v$ is an edge of the perfect matching of $T$. This fact leads us to get the following useful lemma. 
Lemma 2.4 Let $T \in \mathcal{T}_{n}^{+}$, and let $s$ be a positive even integer not greater than $n$. Then there exist a vertex $v \in V(T)$ and a subtree $U$ of $T$ such that

\section{U has a perfect matching;}

2. either $U$ is a component of $T-v$ when $v \notin V(U)$, or $U-v$ is a component of $T-v$ when $v \in V(U)$;

3. $|V(U)| \leq \max \{n-s, s\}$;

4. all components of $(T-V(U))-v$ have order at most $s$, and all but at most one of them have a perfect matching.

Proof: Let $M$ be a perfect matching of $T$. By Lemma 2.3 there exists a vertex $v \in V(T)$ such that one component $T^{\prime}$ of $T-v$ has order $\left|V\left(T^{\prime}\right)\right| \leq \max \{n-1-s, s\}$, and all other components of $T-v$ have orders not exceeding $s$. We know that only one component, say $T_{0}$, has no perfect matching and all the others have perfect matchings.

Suppose $T^{\prime} \neq T_{0}$. Then $M \cap E\left(T^{\prime}\right)$ is a perfect matching of $T^{\prime}$. Since $s$ and $n$ are even, $\left|V\left(T^{\prime}\right)\right| \leq$ $\max \{n-2-s, s\}$. Let $U=T^{\prime}$. Then $T_{0}$ is a component of $(T-V(U))-v$ and its matching number is $\mu\left(T_{0}\right)=\frac{1}{2}\left(V\left(T_{0}\right)-1\right)$. Let $w$ be the only unsaturated vertex of $T_{0}$ which is adjacent with $v$ in $T$ and $w v$ is an edge of $M$. Now let $T_{0}^{\prime}$ be the tree obtained from $T_{0}$ by joining a pendant vertex $u$ to $w$. Actually, we can view this vertex $u$ as the removed vertex $v$. Obviously, $T_{0}^{\prime}$ has a perfect matching $\left(E\left(T_{0}\right) \cap M\right) \cup\{v w\}$ and order $\left|V\left(T_{0}^{\prime}\right)\right|=\left|V\left(T_{0}\right)\right|+1 \leq s$, and $T_{0}^{\prime}$ is a subtree of $T$.

Suppose $T^{\prime}=T_{0}$. Then $T^{\prime}$ has a maximum matching $M_{1}=E\left(T^{\prime}\right) \cap M$ and its matching number is $\mu\left(T^{\prime}\right)=\frac{1}{2}\left(\left|V\left(T^{\prime}\right)\right|-1\right)$. Since $s$ is even, $\left|V\left(T^{\prime}\right)\right| \leq \max \{n-1-s, s-1\}$. Let $w \in V\left(T^{\prime}\right)$ be the only $M_{1}$-unsaturated vertex. Then $w v$ is an edge of $M$. Let $U$ be the tree obtained from $T^{\prime}$ by joining a pendant vertex $u$ to $w$. Actually, we can view this vertex $u$ as the removed vertex $v$. Then $U$ is a subtree of $T$ and is of order not greater than $\max \{n-s, s\}$. Clearly, $M_{1} \cup\{v w\}$ is a perfect matching of $U$ and $U-v=T^{\prime}$.

Lemma 2.5 Let $T$ be a tree in $\mathcal{T}_{2 p}^{+}$. Then for any positive integer $k$ with $1 \leq k \leq p$, there exists a $(k-1)$-vertex subset $V^{\prime} \subset V(T)$ such that all components of $T-V^{\prime}$ have the largest eigenvalues not greater than $\lambda_{1}\left(T_{2 t}^{1}\right)$, where $T_{2 t}^{1}$ is the tree shown in Fig. 1.1 and $t=\lceil p / k\rceil$.

Proof: When $k=1$, the result is actually Theorem 1.3 So we may assume that $k \geq 2$. Let $s=2 t=$ $2\lceil p / k\rceil$, and $T_{0}=T, n_{0}=2 p$. Since $k \geq 2$, we have $n_{0}>s$. We perform the following procedure:

By Lemma 2.4, there are a vertex $v_{1} \in V(T)$ and a subtree $T_{1}$ of order not greater than $\max \left\{n_{0}-s, s\right\}$ such that $T_{1}$ has a perfect matching, $T_{1}-v_{1}$ is a component of $T-v_{1}$ and the other components of $T-v_{1}$ have orders not greater than $s$. Note that $v_{1}$ may not be a vertex of $T_{1}$.

Let $n_{1}=\left|V\left(T_{1}\right)\right|$. If all components of $T-v_{1}$ and $T_{1}$ are of orders not greater than $s$, then we stop the procedure. If not, then $n_{1}>s$. By applying Lemma 2.4 to $T_{1}$ there are a vertex $v_{2} \in V\left(T_{1}\right)$ and a subtree $T_{2}$ of $T_{1}$ such that the order of $T_{2}$ is not greater than $\max \left\{n_{1}-s, s\right\}, T_{2}$ has a perfect matching, $T_{2}-v_{2}$ is a component of $T_{1}-v_{2}$ and the other components of $T_{1}-v_{2}$ have orders not greater than $s$.

Let $n_{2}=\left|V\left(T_{2}\right)\right|$. If all components of $T-\left\{v_{1}, v_{2}\right\}$ and $T_{2}$ are of orders not greater than $s$, then we stop the procedure. If not, we continue to perform the above procedure. Since $n_{0}$ is finite, there are $h$ subtrees $T_{0} \supset T_{1} \supset \cdots \supset T_{h}$ and vertices $v_{1}, \ldots, v_{h}$ (not necessary distinct) such that all components of 
$T-\left\{v_{1}, v_{2}, \ldots, v_{h}\right\}$ are of orders not greater than $s, n_{i}=\left|V\left(T_{i}\right)\right| \leq \max \left\{n_{i-1}-s, s\right\}$ and $v_{i} \in V\left(T_{i-1}\right)$ for $1 \leq i \leq h$. Hence we have $n_{i}>s$ for $1 \leq i \leq h-1$. Since $s=2\lceil p / k\rceil$,

$$
k s=2 k\lceil p / k\rceil \geq 2 k(p / k)=2 p .
$$

Since $n_{i} \leq n_{i-1}-s,(i=1,2, \ldots, h)$,

$$
n_{h-1}-n_{0}=\sum_{i=1}^{h-1}\left(n_{i}-n_{i-1}\right) \leq-(h-1) s .
$$

Hence

$$
s<n_{h-1} \leq 2 p-(h-1) s \leq k s-h s+s=(k-h+1) s .
$$

Thus $h \leq k-1$.

Now we may choose a $(k-1)$-vertex subset $V^{\prime}$ containing $\left\{v_{1}, v_{2}, \ldots, v_{h}\right\}$ such that the components of $T-V^{\prime}$ are of orders not exceeding $s$. By Lemma 2.2 and Theorem 1.3, all components of $T-V^{\prime}$ have their largest eigenvalues not great than $\lambda_{1}\left(T_{2 t}^{1}\right)$. The proof is completed.

Combining Lemma 2.5 with the Cauchy Interlacing Theorem, we obtain the following main result.

Theorem 2.6 Let $T$ be a tree in $\mathcal{T}_{2 p}^{+}$. Then for any positive integer $k$ with $1 \leq k \leq p$, we have

$$
\lambda_{k}(T) \leq \frac{1}{2}\left(\sqrt{\left\lceil\frac{p}{k}\right\rceil-1}+\sqrt{\left\lceil\frac{p}{k}\right\rceil+3}\right)
$$

and this upper bound is best possible when $k=1$.

Proof: Suppose that $T \in \mathcal{T}_{2 p}^{+}$. By Lemma 2.5 , we have a $(k-1)$-vertex subset $V^{\prime} \subset V(T)$ such that all components, say $T_{1}, T_{2}, \ldots, T_{q}$, of $T-V^{\prime}$ are trees with the largest eigenvalues not exceeding $\lambda_{1}\left(T_{2 t}^{1}\right)$, $t=\left\lceil\frac{p}{k}\right\rceil$. By Theorems 2.1 and 1.3 , we obtain

$$
\begin{aligned}
\lambda_{k}(T) & \leq \lambda_{1}\left(T-V^{\prime}\right)=\max _{1 \leq i \leq q} \lambda_{1}\left(T_{i}\right) \\
& \leq \max _{1 \leq i \leq s} \frac{1}{2}\left(\sqrt{\frac{\left|V\left(T_{i}\right)\right|}{2}-1}+\sqrt{\frac{\left|V\left(T_{i}\right)\right|}{2}+3}\right) \\
& \leq \frac{1}{2}\left(\sqrt{\left\lceil\frac{p}{k}\right\rceil-1}+\sqrt{\left\lceil\frac{p}{k}\right\rceil+3}\right)
\end{aligned}
$$

This proves the upper bound 2.1. Obviously, for $k=1$, 2.1) is just the upper bound $\lambda_{1}(T) \leq$ $\frac{1}{2}(\sqrt{p-1}+\sqrt{p+3})$ in Theorem 1.3 and it is best possible upper bound.

Example 2.1 For any $T \in \mathcal{T}_{10}^{+}$, from Theorem 2.6 we get that $\lambda_{1}(T) \leq \frac{1}{2}(\sqrt{4}+\sqrt{8}) \approx 2.414, \lambda_{2}(T) \leq$ $\frac{1}{2}(\sqrt{2}+\sqrt{6}) \approx 1.932, \lambda_{3}(T) \leq \frac{1}{2}(1+\sqrt{5}) \approx 1.618, \lambda_{4}(T) \leq \frac{1}{2}(1+\sqrt{5}) \approx 1.618$ and $\lambda_{5}(T) \leq 1$. We find that $\lambda_{1}(T)$ and $\lambda_{5}(T)$ are tight, which can be verified by the table of the spectra of all trees with $n$ vertices $(2 \leq n \leq 10)$ in $[6]$. 
On the $k^{\text {th }}$ Eigenvalues of Trees with Perfect Matchings

There is a relationship between the characteristic polynomial $P\left(G \odot N_{s} ; \lambda\right)$ of $G \odot N_{s}$ and that of $G$ as follows.

Lemma 2.7 ([6]) $P\left(G \odot N_{s} ; \lambda\right)=\lambda^{p s} P\left(G ; \lambda-\frac{s}{\lambda}\right)$.

Let $\mathcal{T}_{2 p}^{*}$ be the set of the coronas of trees of order $p$ with $N_{1}$, i.e.,

$$
\mathcal{T}_{2 p}^{*}=\left\{T \odot N_{1} \mid T \in \mathcal{T}_{p}\right\}
$$

Obviously, any graph in $\mathcal{T}_{2 p}^{*}$ is a tree and has a perfect matching. Thus we have $\mathcal{T}_{2 p}^{*} \subset \mathcal{T}_{2 p}^{+}$. Note that for any $T^{*} \in \mathcal{T}_{2 p}^{*}$, there is a unique tree $T$ with $T^{*}=T \odot N_{1}$. The tree $T$ is called the contracted tree of the tree $T^{*}$. Now we prove an upper bound on the $k$ th eigenvalue of trees in $\mathcal{T}_{2 p}^{*}$.

Lemma 2.8 Let $T^{*} \in \mathcal{T}_{2 p}^{*}$ and let $T$ be the contracted tree of $T^{*}$. Then

$$
\lambda_{k}\left(T^{*}\right)=\frac{1}{2}\left(\sqrt{\lambda_{k}(T)^{2}+4}+\lambda_{k}(T)\right) .
$$

Proof: By Lemma 2.7, we have $P\left(T^{*} ; \lambda\right)=\lambda^{p} P\left(T ; \lambda-\frac{1}{\lambda}\right)$. Since $\lambda_{k}(T)$ is the $k$ th eigenvalue of $T$ for $k=1,2, \ldots, p$,

$$
P\left(T^{*} ; \lambda\right)=\lambda^{p} \prod_{i=1}^{p}\left(\lambda-\frac{1}{\lambda}-\lambda_{i}(T)\right)=\prod_{i=1}^{p}\left(\lambda^{2}-\lambda_{i}(T) \lambda-1\right) .
$$

So the positive eigenvalues of $T^{*}$ are $\frac{1}{2}\left(\sqrt{\lambda_{i}(T)^{2}+4}+\lambda_{i}(T)\right), i=1,2, \ldots, p$. Since $f(x)=$ $\frac{1}{2}\left(\sqrt{x^{2}+4}+x\right)$ is an increasing function of the variable $x$, the result follows immediately.

Theorem 2.9 Let $T^{*}$ be a tree in $\mathcal{T}_{2 p}^{*}$. Then

$$
\lambda_{k}\left(T^{*}\right) \leq \frac{1}{2}\left(\sqrt{\left\lfloor\frac{p}{k}\right\rfloor-1}+\sqrt{\left\lfloor\frac{p}{k}\right\rfloor+3}\right)
$$

for $k=1,2, \ldots, p$. Moreover, this upper bound is best possible when $k=1$ or $p \not \equiv 0(\bmod k)$.

Proof: Suppose that $T$ is the contracted tree of the tree $T^{*}$. Then $T$ is a tree of order $p$. By Theorem 1.2 , Lemma 2.8 and its proof, we have

$$
\begin{aligned}
\lambda_{k}\left(T^{*}\right) & =\frac{1}{2}\left(\sqrt{\lambda_{k}(T)^{2}+4}+\lambda_{k}(T)\right) \\
& \leq \frac{1}{2}\left(\sqrt{\left\lfloor\frac{p}{k}\right\rfloor-1}+\sqrt{\left\lfloor\frac{p}{k}\right\rfloor+3}\right)
\end{aligned}
$$

for $k \leq\left\lfloor\frac{p}{2}\right\rfloor$. For $k>\left\lfloor\frac{p}{2}\right\rfloor$, since $\lambda_{k}(T) \leq 0$, we have $\lambda_{k}\left(T^{*}\right) \leq 1$. The Equation 2.2 holds, since the right hand side of (2.2) is equal to 1 .

When $k=1$, it is known that this bound is best possible. To show tightness for $k \geq 2$ and $p \not \equiv 0$ $(\bmod k)$, we shall construct a corona of a tree with $N_{1}$. First, we write $p=\lfloor p / k\rfloor k+r$, where $1 \leq r \leq$ $k-1$. Set $t=2\lfloor p / k\rfloor$ and thus $2 p=t k+2 r$. Let $T$ be the tree obtained by joining edges from the 


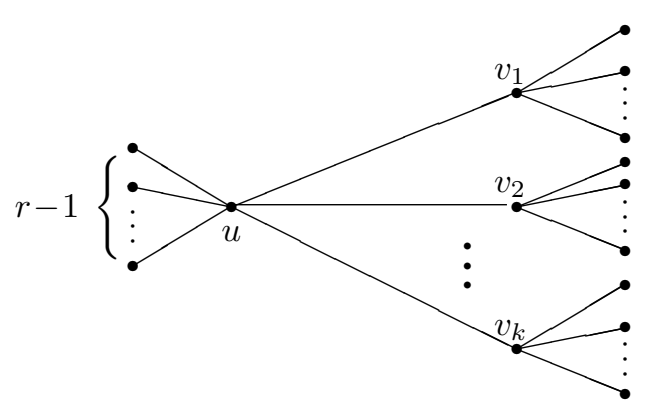

Fig. 2.1: A tree $T$ in the proof of Theorem 2.9

center $u$ of a star $K_{1, r-1}$ to the centers $v_{1}, v_{2}, \ldots, v_{k}$ of $k$ disjoint stars $K_{1, \frac{t}{2}-1}$ (see Fig. 2.1p. Then let $T^{*}=T \odot N_{1} \in \mathcal{T}_{2 p}^{*}$. It is easy to see that

$$
\lambda_{1}(T-u)=\lambda_{2}(T-u)=\cdots=\lambda_{k}(T-u)=\lambda_{1}\left(K_{1, \frac{t}{2}-1}\right)=\sqrt{\frac{t}{2}-1} .
$$

By Lemma 2.2 and the Cauchy Interlacing Theorem we have

$$
\lambda_{k}(T-u) \leq \lambda_{k}(T) \leq \lambda_{k-1}(T-u) .
$$

Therefore,

$$
\lambda_{k}(T)=\sqrt{\frac{t}{2}-1}
$$

By Lemma 2.8 , we have

$$
\lambda_{k}\left(T^{*}\right)=\frac{1}{2}\left(\sqrt{\frac{t}{2}-1}+\sqrt{\frac{t}{2}+3}\right)=\frac{1}{2}\left(\sqrt{\left\lfloor\frac{p}{k}\right\rfloor-1}+\sqrt{\left\lfloor\frac{p}{k}\right\rfloor+3}\right) .
$$

This shows that the upper bound 2.2 is best possible when $p \not \equiv 0(\bmod k)$.

Example 2.2 For any tree $T^{*} \in \mathcal{T}_{10}^{*}$, by Theorem 2.6 we have $\lambda_{1}\left(T^{*}\right) \leq \frac{1}{2}(\sqrt{4}+\sqrt{8}) \approx 2.414, \lambda_{2}\left(T^{*}\right) \leq$ $\frac{1}{2}(1+\sqrt{5}) \approx 1.618, \lambda_{3}\left(T^{*}\right) \leq 1, \lambda_{4}\left(T^{*}\right) \leq 1$ and $\lambda_{5}\left(T^{*}\right) \leq 1$. It can be verified from the table of the spectra of all trees with $n$ vertices $(2 \leq n \leq 10)$ in $[6]$ that these bounds are tight.

Example 2.3 For any tree $T^{*} \in \mathcal{T}_{8}^{*}$, by Theorem 2.6 we have $\lambda_{1}\left(T^{*}\right) \leq \frac{1}{2}(\sqrt{3}+\sqrt{7}) \approx 2.189, \lambda_{2}\left(T^{*}\right) \leq$ $\frac{1}{2}(1+\sqrt{5}) \approx 1.618, \lambda_{3}\left(T^{*}\right) \leq 1$ and $\lambda_{4}\left(T^{*}\right) \leq 1$. We know that the upper bounds on $\lambda_{1}$ and $\lambda_{3}$ are tight but on $\lambda_{2}$ and $\lambda_{4}$ they are not. Actually, the maximum values of $\lambda_{2}$ and $\lambda_{4}$ are approximately 1.356 and 0.477 , respectively. 


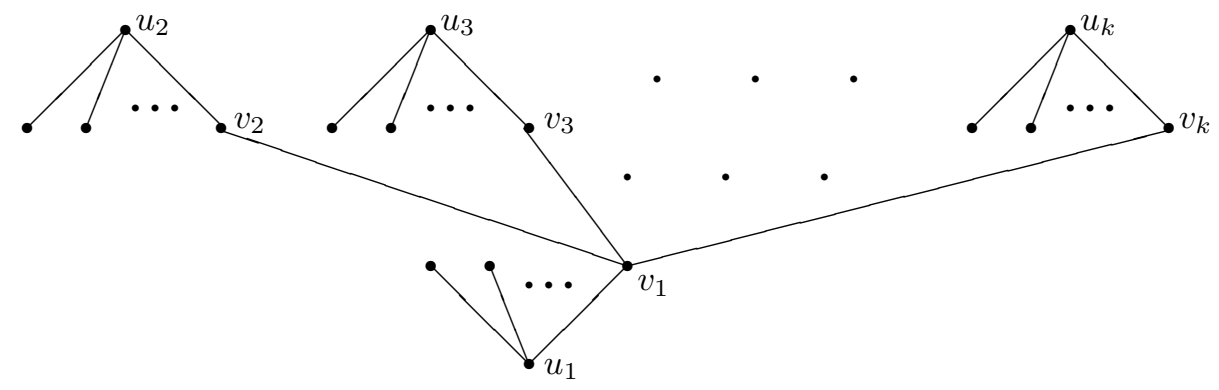

Fig. 2.2: $T$ he tree $T$ in Lemma 2.10

Example 2.3 shows that for those $k$ satisfying $p \equiv 0(\bmod k)$, we usually only have

$$
\lambda_{k}\left(T^{*}\right)<\frac{1}{2}\left(\sqrt{\left\lfloor\frac{p}{k}\right\rfloor-1}+\sqrt{\left\lfloor\frac{p}{k}\right\rfloor+3}\right)
$$

and especially, the upper bound is not as good as that in Theorem 1.2 when $k=2$. However, the upper bound in Theorem 2.6 will be shown to be asymptotically tight when $p \equiv 0(\bmod k)$.

Lemma $2.10([14])$ Let $v$ be a vertex of $G$, and $\mathcal{C}(v)$ be the set of all cycles containing $v$. Then the characteristic polynomial of $G$ satisfies

$$
P(G ; \lambda)=\lambda P(G-v ; \lambda)-\sum_{u v \in E(G)} P(G-v-u ; \lambda)-2 \sum_{Z \in \mathcal{C}(v)} P(G-V(Z) ; \lambda) .
$$

We first take $k$ copies of the star $K_{1, t-1}$ (say $S_{1}, S_{2}, \ldots, S_{k}$ ) with centers $u_{1}, u_{2}, \ldots, u_{k}$, respectively, and choose $v_{i} \in V\left(S_{i}\right) \backslash\left\{u_{i}\right\}(i=1,2, \ldots, k)$. Then add the edges $v_{1} v_{i}(i=2,3, \ldots, k)$ to obtain tree $T$ with $t k$ vertices as shown in Fig. 2.2

The next lemma follows by direct calculation from Lemma 2.10 with $v=v_{1}$, observing that the last term in the lemma becomes zero because there are no cycles containing $v_{1}$.

Lemma 2.11 ([15]) Denoting $f(x)=x^{3}+(t-k) x^{2}-2(k-1) x-(k-1)$, the characteristic polynomial of the tree $T$ shown in Fig. 2.2 is

$$
P(T ; \lambda)=\lambda^{t k-2(k+1)}\left(\lambda^{2}-t+1\right)^{k-2} f\left(\lambda^{2}-t+1\right)
$$

and the kth eigenvalue of $T$ satisfies

$$
\lambda_{k}(T)=\sqrt{t-1+\lambda_{2}(f)}>\sqrt{t-1-\sqrt{\frac{k-1}{t-k}}},
$$

where $\lambda_{2}(f)$ is the second largest root of the equation $f(x)=0$. 
Denote the maximum value of the $k$ th largest eigenvalue of the trees in $\mathcal{T}_{2 p}^{*}$ by $\lambda_{k}^{*}(2 p)$. Then Theorem 2.9 tells us that $\lambda_{k}^{*}(2 p) \leq \frac{1}{2}\left(\sqrt{\left\lfloor\frac{p}{k}\right\rfloor-1}+\sqrt{\left\lfloor\frac{p}{k}\right\rfloor+3}\right)$. We shall give a lower bound for $\lambda_{k}^{*}(2 p)$, which shows that as $k$ gets large, the upper bound in Theorem 2.6 is asymptotically tight for the value of $\lambda_{k}^{*}(2 p)$ when $p \equiv 0(\bmod k)$.

Theorem 2.12 Let $p$ and $k$ be integers with $1 \leq k \leq p$. If $t=\left\lfloor\frac{p}{k}\right\rfloor>k$, then

$$
\lambda_{k}^{*}(2 p)>\frac{1}{2}\left(\sqrt{\left\lfloor\frac{p}{k}\right\rfloor-1-\sqrt{\frac{k-1}{t-k}}}+\sqrt{\left\lfloor\frac{p}{k}\right\rfloor+3-\sqrt{\frac{k-1}{t-k}}}\right) .
$$

Proof: Let $T^{*}=T \odot N_{1} \in \mathcal{T}_{2 t k}^{*}$ by taking the tree $T$ with $t k$ vertices described in Fig. 2.2 From Lemma 2.11, it is easy to see that the second largest root $\lambda_{2}(f)$ of $f(x)=0$ is negative. Note that $f(0)=-(k-1)<0, f\left(-\sqrt{\frac{k-1}{t-k}}\right)>0$ and $\lim _{x \rightarrow-\infty} f(x)=-\infty$. So we know that $\lambda_{2}(f)>-\sqrt{\frac{k-1}{t-k}}$. Moreover, the expression $\lambda=\frac{1}{2}(\sqrt{t-1+\alpha}+\sqrt{t+3+\alpha})$ can be regarded as a strictly increasing function of the variable $\alpha$. Thus, by Lemmas 2.8 and 2.11, we have

$$
\begin{aligned}
\lambda_{k}\left(T^{*}\right) & =\frac{1}{2}\left(\sqrt{t-1+\lambda_{2}(f)}+\sqrt{t+3+\lambda_{2}(f)}\right) \\
& >\frac{1}{2}\left(\sqrt{t-1-\sqrt{\frac{k-1}{t-k}}}+\sqrt{t+3-\sqrt{\frac{k-1}{t-k}}}\right) .
\end{aligned}
$$

There is a tree $U$ of order $p$ containing $T$ described above. Hence $U^{*}=U \odot N_{1} \in \mathcal{T}_{2 p}^{*}$ and

$$
\begin{aligned}
\lambda_{k}\left(U^{*}\right) & \geq \lambda_{k}\left(T^{*}\right)>\frac{1}{2}\left(\sqrt{t-1-\sqrt{\frac{k-1}{t-k}}}+\sqrt{t+3-\sqrt{\frac{k-1}{t-k}}}\right) \\
& =\frac{1}{2}\left(\sqrt{\left\lfloor\frac{p}{k}\right\rfloor-1-\sqrt{\frac{k-1}{t-k}}}+\sqrt{\left\lfloor\frac{p}{k}\right\rfloor+3-\sqrt{\frac{k-1}{t-k}}}\right) .
\end{aligned}
$$

Thus we get the theorem.

Remark: If we let $t \rightarrow \infty$ (that is, $2 p \rightarrow \infty$ ) for a fixed $k$, then $\sqrt{\frac{k-1}{t-k}} \rightarrow 0$, i.e.,

$$
\lambda_{k}^{*}(2 p) \rightarrow \frac{1}{2}\left(\sqrt{\left\lfloor\frac{p}{k}\right\rfloor-1}+\sqrt{\left\lfloor\frac{p}{k}\right\rfloor+3}\right) \text { as } t \rightarrow \infty .
$$

So we can say that our upper bound 2.2 is asymptotically tight. Of course, if we denote the maximum value of the $k$ th eigenvalues of trees in $\mathcal{T}_{2 p}^{+}$by $\lambda_{k}^{+}(2 p)$, then by Theorem 2.9. we have $\lambda_{k}^{+}(2 p) \geq$ $\frac{1}{2}\left(\sqrt{\left\lfloor\frac{p}{k}\right\rfloor-1}+\sqrt{\left\lfloor\frac{p}{k}\right\rfloor+3}\right)$. So the upper bound 2.11 is also asymptotically tight in a sense. 
On the $k^{\text {th }}$ Eigenvalues of Trees with Perfect Matchings

\section{References}

[1] N.L. Biggs, Algebraic Graph Theory, 2nd Ed., Cambridge University Press, Cambridge, 1993.

[2] J.A. Bondy and U.S.R. Murty, Graph Theory with Applications, MacMillan Press Ltd., 1976.

[3] A. Chang, Bounds on the second largest eigenvalue of a tree with perfect matchings, Linear Algebra and its Applicatins, 283 (1998), 247-255.

[4] A. Chang, The sublargest value and quasi-smallest value of the largest eigenvalue of the tree with perfect matchings (in Chinese), Applied Mathematics - A Journal of Chinese Universities. 14(4) (1999), 397-403.

[5] A. Chang, On the largest eigenvalue of a tree with perfect matchings, Discrete Mathematics, 269 (2003), 45-63.

[6] D. Cvetković, M. Doob and H. Sachs, Spectra of Graphs -Theory and Applications, Academic Press, New York, 1980.

[7] R. Frucht and F. Harary, On the corona of two graphs, Aequationes Mathematicae, 4 (1970), 322-325.

[8] C.D. Godsil, Inverse of trees, Combinatorica, 5 (1985), 33-39.

[9] J-M. Guo and S-W. Tan, A conjecture on the second largest eigenvalue of a tree with perfect matchings, Linear Algebra and its Applications, 347 (2002), 9-15.

[10] J-M. Guo and S-W. Tan, A note on the second largest eigenvalue of a tree with perfect matchings, Linear Algebra and its Applications, 380 (2004), 125-134.

[11] M. Hofmeister, On the two largest eigenvalues of trees, Linear Algebra and its Applications, 260 (1997), 43-59.

[12] Y. Hong, The $k$ th largest eigenvalue of a tree, Linear Algebra and its Applications, 73 (1986), 151-155.

[13] A. Neumaier, The second largest eigenvalue of a tree, Linear Algebra and its Applications, 46 (1982), 9-25.

[14] A.J. Schwenk, Computing the characteristic polynomial of a graph, In: Graphs and Combinatorics (Lecture Notes in Mathematics, 406), edited by R.A. Bari, F. Harary, Springer-Verlag, New York, 1974, 153-173.

[15] J.Y. Shao, Bounds on the $k$ th eigenvalues of trees and forests, Linear Algebra and its Applications, 149 (1991), 19-34.

[16] J.Y. Shao and Y. Hong, Bounds on the smallest positive eigenvalue of trees with a perfect matching (in Chinese), Science Bulletin, 18 (1991), 1361-1364.

[17] G.H. Xu, On the spectral radius of trees with perfect matchings, in: Combinatorics and Graph Theory, World Scientific, Singapore, 1997.

[18] F. Zhang and A. Chang, Acyclic molecules with greatest HOMO-LUMO separation, Discrete Applied Mathematics, 98 (1999), 165-171. 
